Pacific Journal of Mathematic 


\title{
COALLOCATION BETWEEN LATTICES WITH APPLICATIONS TO MEASURE EXTENSIONS
}

\author{
WILLIAM JOSEPHSON
}

It is well known that in a locally compact Hausdorff space every countably additive measure on $R_{\sigma}\left(\mathscr{K}_{\delta}\right)$, the $\sigma$-ring generated by the compact $G_{\delta}$ sets, can be extended to a countably additive measure on $\sigma(\mathscr{F})$, the $\sigma$-algebra generated by the closed sets. In a locally compact Hausdorff space $\mathscr{F}$, the lattice of closed sets, countably coallocates (Definition 4.7) the lattice of compact $G_{\delta}$ sets. Our purpose is to show that coallocation and countable coallocation are properties basic to many extension theorems.

Dubins [5] considered the following situation. $K \subseteq L$ are two lattices containing the null set (a lattice is a collection of subsets of some set closed under finite unions and intersections). $u$ is a bounded measure defined on $K$. Dubins asked when $u_{*}$, defined by $u_{*}(b)=$ $\sup \{u(k) / k \subseteq b, k \in K\}$, is a measure on $L$. A necessary and sufficient condition is for $L$ to allocate $K$. $L$ allocates $K$ if the following is true. For any $k \in K$ contained in the union of two sets $l$ and $h$ from $L$ there exist sets $p$ and $q$ from the lattice $K$ such that $k=p \cup q$ and $p \subseteq l$, $q \subseteq h$.

With two lattices $K \subseteq L$ and $u$ a measure on $K$, we show that a sufficient condition for $u^{* *}$, defined by $u^{* *}(b)=\inf \left\{u_{*}\left(l^{\prime}\right) / b \subseteq l^{\prime}, l^{\prime}\right.$ $\left.\in L^{\prime}\right\}$, to be a measure on the algebra generated by $L$ is for $u_{*}$ to be modular on $L^{\prime} . \quad l^{\prime}$ is the complement of the set $l$ and $L^{\prime}=\left\{l^{\prime} / l \in L\right\} . \quad$ It follows that if $u$ is a $K$ inner regular measure on $R(K)$, the ring generated by $K$, then $u^{* *}$ is a $L$ inner regular extension of $u$ to $A(L)$, the algebra generated by $L$.

Thus when $L$ coallocates $K$ (i.e. $L^{\prime}$ allocates $K$ ) Dubin's result shows that for every $K$ regular bounded measure $u$ on $R(K), u^{* *}$ is a $L$ regular extension of $u$ to $A(L)$. If $L$ countably coallocates $K$ then $u^{* *}$ is countably additive when $u$ is countably additive. From this we obtain the stated result on locally compact Hausdorff spaces [Halmos 7] as well as a related result by Levin and Stiles [8]. Countable coallocation also yields an extension theorem by Marik [9] on countably paracompact normal spaces and a theorem by Berberian [2]. In most instances we can and do prove our results for measures that are not bounded.

We also look at measures that are $\tau$-smooth. A measure $u$ on $K$ is $\tau$-smooth if for any net $\left\{k_{\alpha}\right\}$ decreasing to $\varnothing, k_{\alpha} \in K, \lim _{\alpha} u\left(k_{\alpha}\right)=0$. We 
show that any bounded $K$ regular measure $u$ on $R(K)$ that is $\tau$-smooth on $K$ can be extended to a bounded measure on $A(\tau(K))$ that is $\tau$-smooth on $\tau(K) . \quad \tau(K)$ is the smallest lattice containing $K$ that is closed under arbitrary intersections. We prove $u_{*}$ is modular on $\tau(K)^{\prime}$ and obtain $u^{* *}$, defined with respect to $\tau(K)$, as the desired extension.

2. Definitions and notation. All lattices are collections of subsets of an abstract set $X$ that are closed under finite unions and intersections. The fact that $X$ contains points has no importance in this paper - the boolean algebra of all subsets of $X$ can be replaced by any complete boolean algebra. Subsets of $X$ will be denoted by lower case letters. If we are considering a lattice $L$ and a set $l$, it will usually be assumed that $l$ belongs to $L$.

$l^{\prime}$ denotes the complement of the set $l$ in $X$ and $L^{\prime}=$ $\left\{l^{\prime} / l \in L\right\} . \quad R(L)$ is the ring generated by $L ; A(L)$ the algebra generated by $L . \quad R_{\sigma}(L)$ is the $\sigma$-ring generated by $L$ and $\sigma(L)$ is the $\sigma$-algebra generated by $L$.

A measure $u$ on a lattice $A$ is an extended real valued set function such that for $a, b \in A$

(i) $u(a)+u(b)=u(a \cup b)+u(a \cap b)$.

(ii) $u(a)+u(b)=u(a \cup b)$ whenever $a \cap b=\varnothing$.

(iii) $a \subseteq b$ implies $u(a) \leqq u(b)$.

Let $K$ be a lattice contained in $A$. A measure $u$ on $A$ is $K$ inner regular if for any $a \in A, u(a)=\sup \{u(k) / k \subseteq a, k \in K\}$.

A measure $u$ on a lattice $A$ is $\sigma$-smooth if for any sequence $\left\{a_{n}\right\}$ decreasing to $\varnothing, \lim _{n} u\left(a_{n}\right)=0 . \quad u$ is countably additive on $A$ if $\Sigma_{1}^{\infty} u\left(a_{n}\right)=u\left(\cup_{1}^{\infty} a_{n}\right)$ whenever $\left\{a_{n}\right\}$ is a disjoint sequence of sets from $A$ such that $\cup_{1}^{\infty} a_{n} \in A$. For a ring $A$ any finite valued measure $u$ which is $\sigma$-smooth on $K \subseteq A$ and $K$ inner regular is countably additive on $A$.

A measure $u$ on a lattice $A$ is $\sigma$-finite if for every $a \in A, a$ is contained in $\bigcup_{1}^{\infty} a_{n}$ where $a_{n} \in A$ and $u\left(a_{n}\right)$ is finite for all $n$. If $A$ is a ring then by the Caratheodory extension theorem any countably additive, $\sigma$-finite measure $u$ on $A$ can be uniquely extended to a countably additive measure on $R_{\sigma}(A)$. The extension is the outer measure defined by $\hat{u}(b)=\inf \left\{\sum_{1}^{\infty} u\left(a_{n}\right) / b \subseteq \bigcup_{1}^{\infty} a_{n}, a_{n} \in A\right\}$.

The bounded measures on the algebra $A(L)$ are denoted by $M(L)$. It is easy to verify that if $u$ is bounded and $L$ inner regular then $u(a)=\inf \left\{u\left(l^{\prime}\right) / a \subseteq l^{\prime}, l \in L\right\}$ for $a \in A(L)$. A measure satisfying the last equality is called $L^{\prime}$ outer regular. If a measure is both $L$ inner regular and $L^{\prime}$ outer regular then it is $L$ regular. The $L$ regular, bounded measures on $A(L)$ are denoted by $M_{r}(L)$. Those measures belonging to $M_{r}(L)$ which are $\sigma$-smooth are denoted by $M_{r}^{s}(L)$. These 
measures are countably additive and hence can be uniquely extended to a countably additive measure on $\sigma(L)$.

For a measure $u$ on a lattice $K$ which contains $\varnothing, u_{*}$ is defined as in the introduction. The definition of $u^{* *}$ as given in the introduction depends on the lattice $L$ used ( $L$ must also contain $\varnothing$ ).

3. The modularity of $u_{*}$. Let $K \subseteq L$ be two lattices containing $\varnothing$ and $u$ a measure on $K . \quad u_{*}$ is modular on $L^{\prime}$ if $u_{*}\left(l_{1}^{\prime}\right)+$ $u_{*}\left(l_{2}^{\prime}\right)=u_{*}\left(l_{1}^{\prime} \cup l_{2}^{\prime}\right)+u_{*}\left(l_{1}^{\prime} \cap l_{2}^{\prime}\right)$. We now show that if $u_{*}$ is modular on $L^{\prime}$ then $u^{* *}$ is an $L^{\prime}$ outer regular measure on $A(L)$ where $u^{* *}$ is defined with respect to $L$. Furthermore $u^{* *}$ is a complete measure on $\mathscr{E}\left(u, L^{\prime}\right)=\left\{e / u^{* *}\left(l^{\prime}\right)=u^{* *}\left(e \cap l^{\prime}\right)+u^{* *}\left(e^{\prime} \cap l^{\prime}\right)\right.$ for all $\left.l \in L\right\}$.

The easy proofs of the following lemmas are omitted.

LeMmA 3.1. Let $u$ be a measure on $K$. If $u_{*}$ is modular on $L^{\prime}$ then for $a, b$ subsets of $X$,

$$
u^{* *}(a \cup b)+u^{* *}(a \cap b) \leqq u^{* *}(a)+u^{* *}(b) .
$$

LeMma 3.2. Let $u$ be a measure on $K$ and $u_{*}$ be modular on $L^{\prime}$. Suppose $l^{\prime} \cap a=\varnothing$, where $a$ is any subset of $X$. Then $u^{* *}\left(l^{\prime}\right)+$ $u^{* *}(a)=u^{* *}\left(a \cup l^{\prime}\right)$.

$u^{*}$ is $\sigma$-smooth on $L^{\prime}$ if $\lim _{n} u_{*}\left(l_{n}^{\prime}\right)=u_{*}\left(\bigcup_{1}^{\infty} l_{n}^{\prime}\right)$ whenever $\left\{l_{n}^{\prime}\right\}$ is an increasing sequence such that $\bigcup_{1}^{\infty} l_{n}^{\prime} \in L^{\prime}$.

THEOREM 3.3. Let $u$ be a measure on $K$.

(i) The modularity of $u_{*}$ on $L^{\prime}$ is equivalent to $u^{* *}$ being an $L^{\prime}$ outer regular measure on $A(L)$.

(ii) If $u_{*}$ is modular on $L^{\prime}$ then $\mathscr{E}\left(u, L^{\prime}\right)$ is an algebra containing $A(L)$ and $u^{* *}$ is a complete measure on $\mathscr{E}\left(u, L^{\prime}\right)$.

(iii) Suppose $L$ is closed under countable intersections. If $u_{*}$ is modular and $\sigma$-smooth on $L^{\prime}$ then $\mathscr{E}\left(u, L^{\prime}\right)$ is a $\sigma$-algebra containing $\sigma(L)$ and $u^{* *}$ is countably additive on $\mathscr{E}\left(u, L^{\prime}\right)$.

Proof. (i), (ii). That modularity is necessary is obvious. The sufficiency of (i) and (ii) will be proved. If $u_{*}$ is modular on $L^{\prime}$ then $\mathscr{E}\left(u, L^{\prime}\right)$ is closed under complementation and by Lemma 3.2 it contains $L^{\prime}$.

Fix $l^{\prime} \in L^{\prime}$. It is sufficient to assume $u_{*}\left(l^{\prime}\right)$ is finite. Let $e_{1}, e_{2}$ belong to $\mathscr{E}\left(u, L^{\prime}\right)$. By Lemma 3.1,

$$
u_{*}\left(l^{\prime}\right) \leqq u^{* *}\left(\left(e_{1} \cup e_{2}\right) \cap l^{\prime}\right)+u^{* *}\left(\left(e_{1} \cup e_{2}\right)^{\prime} \cap l^{\prime}\right) .
$$


For the reverse inequality choose $l_{j}^{\prime}, h_{j}^{\prime}$ from $L^{\prime}$ such that $l_{j}^{\prime} \supseteq e_{j} \cap l^{\prime}$, $h_{l}^{\prime} \supseteq e_{j}^{\prime} \cap l^{\prime}$ and

$$
u_{*}\left(l^{\prime}\right) \geqq u_{*}\left(l_{j}^{\prime}\right)+u_{*}\left(h_{j}^{\prime}\right)-\epsilon / 3 \quad j=1,2 .
$$

We claim that

$$
u_{*}\left(l^{\prime}\right) \geqq u_{*}\left(l_{1}^{\prime} \cup l_{2}^{\prime}\right)+u_{*}\left(h_{1}^{\prime} \cap h_{2}^{\prime}\right)-\epsilon .
$$

This inequality is implied by

$$
u_{*}\left(l_{1}^{\prime} \cup l_{2}^{\prime}\right)+u_{*}\left(h_{1}^{\prime} \cap h_{2}^{\prime}\right) \leqq u_{*}\left(l_{1}^{\prime}\right)+u_{*}\left(h_{1}^{\prime}\right)+\frac{2}{3} \epsilon
$$

which is equivalent to

$$
u_{*}\left(l_{2}^{\prime}\right)+u_{*}\left(h_{2}^{\prime}\right) \leqq u_{*}\left(l_{1}^{\prime} \cap l_{2}^{\prime}\right)+u_{*}\left(h_{1}^{\prime} \cup h_{2}^{\prime}\right)+\frac{2}{3} \epsilon
$$

by the modularity of $u_{*}$. The last inequality is true by (2) and the modularity of $u_{*}$.

(3) implies the reverse direction of (1) and hence $e_{1} \cup e_{2}$ belongs to $\mathscr{E}\left(u, L^{\prime}\right)$. Hence $\mathscr{E}\left(u, L^{\prime}\right)$ is an algebra containing $L$.

To show $u^{* *}$ is a measure suppose $l^{\prime}$ contains $e_{1} \cup e_{2}$ and that $u\left(l^{\prime}\right)-u\left(e_{1} \cup e_{2}\right)<\epsilon$. Then by (3)

$$
u_{*}\left(l_{1}^{\prime}\right)+u_{*}\left(l_{2}^{\prime}\right) \leqq u_{*}\left(l^{\prime}\right)+u_{*}\left(l_{1}^{\prime} \cap l_{2}^{\prime}\right)+\epsilon .
$$

Therefore

$$
u^{* *}\left(e_{1}\right)+u^{* *}\left(e_{2}\right) \leqq u^{* *}\left(e_{1} \cup e_{2}\right)+u^{* *}\left(e_{1} \cap e_{2}\right)+2 \epsilon
$$

By Lemma $3.1 u^{* *}$ is modular on $\mathscr{E}\left(u, L^{\prime}\right)$ and by Lemma $3.2 u^{* *}(\varnothing)=$ 0 . It is easy to verify that $\mathscr{E}\left(u, L^{\prime}\right)$ contains all $e$ such that $u^{* *}(e)=0$.

(iii) Let $\left\{e_{n}\right\}$ be a sequence from $\mathscr{E}\left(u, L^{\prime}\right)$. Choose $l_{1}^{\prime} \supseteq e_{n} \cap l^{\prime}$, $b_{n}^{\prime} \supseteq e_{n} \cap l^{\prime}$ such that

$$
u_{*}\left(l^{\prime}\right) \geqq u_{*}\left(l_{n}^{\prime}\right)+u_{*}\left(h_{n}^{\prime}\right)-\epsilon / 2^{n}
$$

We can show using (4) and the modularity of $u_{*}$ that

$$
u_{*}\left(\bigcup_{1}^{n} l_{l}^{\prime}\right)+u_{*}\left(\bigcap_{1}^{n} h_{j}^{\prime}\right) \leqq u_{*}\left(l^{\prime}\right)+\sum_{1}^{n} \frac{\epsilon}{2^{j}} .
$$

Since $u_{*}$ is $\sigma$-smooth on $L^{\prime}$ there exists an $n$ large enough such that 


$$
u_{*}\left(l^{\prime}\right) \geqq u_{*}\left(\bigcup_{1}^{\infty} l_{1}^{\prime}\right)+u_{*}\left(\bigcap_{1}^{n} h_{1}^{\prime}\right)-2 \epsilon
$$

It follows that

$$
u_{*}\left(l^{\prime}\right) \geqq u^{* *}\left(l^{\prime} \cap\left(\bigcup_{1}^{\infty} e_{l}\right)\right)+u^{* *}\left(l^{\prime} \backslash\left(\bigcup_{1}^{\infty} e_{l}\right)\right)-2 \epsilon .
$$

Therefore by Lemma $3.1, \bigcup_{1}^{\infty} e_{j} \in \mathscr{E}\left(u, L^{\prime}\right)$.

To show $u^{* *}$ is countably additive, we can assume $\sum_{1}^{\infty} u^{* *}\left(e_{J}\right)$ is finite. Choose $f_{j}^{\prime} \supseteq e_{j}, f_{j}^{\prime} \in L^{\prime}$ such that $u_{*}\left(f_{j}^{\prime}\right)-u^{* *}\left(e_{j}\right) \leqq \epsilon / 2^{\prime}$. Let $l^{\prime}=\bigcup_{1}^{\infty} f_{j}^{\prime}$. Then since $u_{*}$ is $\sigma$-smooth and modular on $L^{\prime}$,

$$
u_{*}\left(l^{\prime}\right) \leqq \sum_{1}^{\infty} u_{*}\left(f_{j}^{\prime}\right)<+\infty
$$

Inequality (7) holds for $l^{\prime}$ and since $l^{\prime} \supseteq \bigcup_{1}^{\infty} e_{j}$,

$$
\begin{aligned}
u^{* *}\left(\bigcup_{1}^{\infty} e_{j}\right) & \leqq u_{*}\left(l^{\prime}\right) \\
& \leqq \sum_{1}^{\infty} u_{*}\left(f_{j}^{\prime}\right) \\
& \leqq \sum_{1}^{\infty} u^{* *}\left(e_{j}\right)+2 \epsilon .
\end{aligned}
$$

Thus $u^{* *}$ is countably additive on $\mathscr{E}\left(u, L^{\prime}\right)$.

We now give sufficient conditions for $u^{* *}$ to extend $u$.

THEOREM 3.4. Let $u$ be a $K$ inner regular measure on $S(K)$ which represents either $A(K)$ or $R(K)$. If $u_{*}$ is modular on $L^{\prime}$ and $u^{* *}$ is finite on $K$ then $u=u^{* *}$ on $S(K)$.

Proof. $u(b)=u^{* *}(b)$ when $u(b)=+\infty$. If $u(b)$ is finite then $u^{* *}(b)$ is finite. This follows because every $b \in S(K)$ is of the form $\bigcup_{j=1}^{n} k_{j} \cap h_{j}^{\prime}$ where for all $j, h_{j} \in K$ and either $k_{j} \in K$ or $k_{\jmath}=X$.

Choose $l^{\prime} \supseteq b$ such that $u_{*}\left(l^{\prime}\right)-u^{* *}(b) \leqq \epsilon / 3$. Choose $k_{0} \subseteq b$ such that $u(b)-u\left(k_{0}\right) \leqq \epsilon / 3$ and choose $k_{1} \subseteq l^{\prime}$ such that $u_{*}\left(l^{\prime}\right)-u\left(k_{1}\right) \leqq \epsilon / 3$. Let $k=k_{0} \cup k_{1}$. Then since $u$ is $K$ inner regular, $|u(k)-u(b)| \leqq \frac{2}{3} \epsilon$. Then $u_{*}\left(l^{\prime}\right)-u(b)<\epsilon$. Hence $u^{* *}(b)=u(b)$.

A set function $v$ on a collection of subsets $\mathscr{H}$ is $\sigma$-finite with respect to $\mathscr{S} \subset \mathscr{H}$ if for every $h \in \mathscr{H}, h \subseteq \bigcup_{1}^{\infty} s_{j}$ where $s_{j} \in \mathscr{S}$ and $v\left(s_{j}\right)$ is finite for 
all $j$. Note that since our measure $u$ is finite on $K, u$ is $\sigma$-finite with respect to $R(K)$ when $u$ is defined on $R_{\sigma}(K)$.

THEOREM 3.5. Suppose $L$ is closed under countable intersections. Suppose $u_{*}$ is modular and $\sigma$-smooth on $L^{\prime}$ where $u$ is a countably additive measure defined on

(i) $\sigma(K), \sigma$-finite with respect to $A(K)$. If $u^{* *}$ is finite on $K$ then it is a countably additive extension of $u$ to $\sigma(L)$.

(ii) $R_{\sigma}(K)$. If $u^{* *}$ is finite valued on $K$ then $u^{* *}$ is a countably additive extension of $u$ to $\sigma(L)$.

Proof. If $u$ is a countably additive, $\sigma$-finite measure on a $\sigma$-ring generated by a ring $S$ then for all $b$ in the $\sigma$-ring,

$$
u(b)=\inf \left\{\sum_{1}^{\infty} u\left(s_{j}\right) / b \subset \bigcup_{1}^{\infty} s_{j}, s_{j} \in S\right\} .
$$

That $u=u^{* *}$ follows in both cases from this and Theorem 3.4.

Define $K \cap L=\{k \cap l / k \in K, l \in L\}$.

Corollary 3.6. Suppose $u \in M_{r}(K)$ and $u_{*}$ modular on $L^{\prime}$. Then $u^{* *}$ is a $K \cap L$ regular extension of $u$ to $A(L)$. If $u$ is countably additive, $u_{*} \sigma$-smooth on $L^{\prime}$, and L closed under countable intersections then $u^{* *}$ is a countably additive measure on $\sigma(L)$.

\section{Coallocation and the extension of $K$ inner regular} measures. We will assume throughout this section that $K \subseteq L$, and that any measure on $K$ (or $A(K), R(K), \sigma(K), R_{\sigma}(K)$ ) is finite valued on $K$. In most examples we consider there should be no confusion as to which lattice is used for $L$ in the definition of $u^{* *}$. We specify this lattice only occasionally.

Allocation is defined as in the introduction. A lattice $L$ coallocates $K$ if $L^{\prime}$ allocates $K$. Though Dubin's paper deals with bounded measures on a lattice, we state his theorem for any extended real valued measure. His proof remains valid despite the change.

THEOREM 4.1. Let $\varnothing \in H$; and let $J$ be any other lattice (J need not contain $\varnothing)$. The following two statements are equivalent.

(i) For every measure $u$ on $H, u_{*}$ is a measure on $J$.

(ii) $J$ allocates $H$.

Proof. Assume $J$ allocates $H$. Choose from $J$ any $j_{1}, j_{2}$ and choose from $H h \subseteq j_{1} \cup j_{2}, l \subseteq j_{1} \cap j_{2}$. Then since $J$ allocates $H$ there exists 
$p_{1}, p_{2} \in H$ such that

(1) $p_{1} \subseteq j_{1}, p_{2} \subseteq j_{2}$

(2) $p_{1} \cup p_{2}=h \cup l, p_{1} \cap p_{2} \supseteq l$.

Therefore $u_{*}\left(j_{1} \cup j_{2}\right)+u_{*}\left(j_{1} \cap j_{2}\right) \leqq u_{*}\left(j_{1}\right)+u_{*}\left(j_{2}\right)$. The reverse inequality is always true. Thus (ii) implies (i).

Assuming $J$ does not allocate $H$, Dubins constructed a measure $u$ on $H$ for which $u_{*}$ is not a measure on $J$. Thus (i) implies (ii).

Corollary 4.2. Suppose L coallocates $K$ and $u \in M(K)$. Define $u^{* *}$ with respect to $L$.

(i) $u^{* *}$ is a complete measure on $\mathscr{E}\left(u, L^{\prime}\right) \supseteq A(L)$ and is the smallest $L^{\prime}$ outer regular measure on $\mathscr{E}\left(u, L^{\prime}\right)$ such that $u^{* *} \geqq u$ on $K$.

(ii) If $u \in M_{r}(K)$ then $u^{* *} \in M_{r}(L)$ and $u^{* *}=u$ on $A(K)$.

(iii) If $u \in M_{r}^{s}(K), u_{*} \sigma$-smooth on $L^{\prime}$ and $L$ closed under countable intersections then $u^{* *} \in M_{r}^{s}(L)$ and $u^{* *}=u$ on $A(K)$.

For any lattice $K, R(K)$ is an ideal in $A(K)$, i.e. $r \cap a$ belongs to $R(K)$ whenever $r \in R(K), a \in A(K)$. Thus $A(K)$ coallocates $R(K)$. Hence for any $K$ inner regular measure $u$ on $R(K), u^{* *}$ defined with respect to $A(K)$ is an extension of $u$ to $A(K)$. Since $u^{* *}=u_{*}$, the extension is $K$ inner regular.

In many instances the lattice $K^{\prime}$ separates the lattice $L$. A lattice $H$ separates $L$ if whenever $l_{1} \cap l_{2}=\varnothing$, there exists disjoint sets $h_{1}, h_{2}$ such that $h_{1} \supseteq l_{1}, h_{2} \supseteq l_{2}$. $H$ coseparates $L$ if $H^{\prime}$ separates $L$.

THEOREM 4.3. Suppose $K$ coseparates $L$ and $K \subseteq L$. Then $L$ coallocates $K$.

Proof. Suppose $l_{1}^{\prime} \cup l_{2}^{\prime} \supseteq k$. Then $l_{1} \cap k, l_{2} \cap k$ are disjoint members of $L$. Since $K$ coseparates $L$ there exist disjoint sets $k_{1}^{\prime}, k_{2}^{\prime}$ containing $l_{1} \cap k$ and $l_{2} \cap k$ respectively. Since $k_{1} \subseteq k^{\prime} \cup l_{1}^{\prime}, k_{1} \cap k \subseteq l_{1}^{\prime}$. Similarly $k_{2} \cap k \subseteq l_{2}^{\prime}$. Now $\left(k_{2} \cap k\right) \cup\left(k_{1} \cap k\right)=k$.

Let $X$ be a topological space. We give the following notation for some natural lattices occuring in $X . \quad \mathscr{F}$ is the lattice of closed sets, $\mathscr{Z}$ is the lattice of zero sets, $\mathscr{K}$ is the lattice of compact sets, and $\mathscr{K}_{\delta}$ is the lattice of compact $G_{\delta}$ sets. If $X$ is a normal space then $\mathscr{Z}$ coseparates $\mathscr{F}$ by Urysohn's lemma. Hence every $u \in M_{r}(\mathscr{Z})$ extends to $u^{* *} \in M_{r}(\mathscr{F})$.

$\mathscr{F}$ coseparates itself in a normal space and $\mathscr{Z}$ coseparates itself in an arbitrary topological space. Consequently for any $u \in M(\mathscr{Z})$, in any space $X, u^{* *}$ is the smallest outer regular measure on $A(\mathscr{Z})$ such that $u^{* *} \geqq u$. Here $u^{* *}$ is defined with respect to $\mathscr{Z}$. 
It will follow from the next theorem that $\mathscr{F}$ coallocates $\mathscr{K}_{\delta}$ in any completely regular Hausdorff space.

Definition 4.4. A lattice $K$ is an $L$-ideal if $K \cap L \subseteq K . \quad K \cap L=$ $\{k \cap l / k \in K, l \in L\}$.

THEOREM 4.5. Let $K$ be an $H$-ideal where $K \subseteq H \subseteq L$. If $H$ coseparates $K \cap L$ then $L$ coallocates $K$.

Proof. Let $l_{1}^{\prime} \cup l_{2}^{\prime} \supseteq k$. Then $\left(k \cap l_{1}\right) \cap\left(k \cap l_{2}\right)=\varnothing$. There exists $h_{1}^{\prime}$ and $h_{2}^{\prime}$ which are disjoint and contain $k \cap l_{1}$, and $k \cap l_{2}$ respectively. Then $h_{1} \cap k \subseteq l_{1}^{\prime}, h_{2} \cap k \subseteq l_{2}^{\prime}$ and $\left(h_{1} \cup h_{2}\right) \cap k=k$. Since $K$ is an $H$ ideal, $L$ coallocates $K$.

In a completely regular Hausdorff space $\mathscr{K}_{\delta}$ is a $\mathscr{Z}$-ideal. $\mathscr{Z}$ coseparates the compact sets and therefore $\mathscr{Z}$ certainly coseparates $\mathscr{K}_{\delta} \cap \mathscr{F}$. Hence $\mathscr{F}$ coallocates $\mathscr{K}_{\delta}$. Therefore we have the following.

THEOREM 4.6. Let $X$ be a completely regular Hausdorff space. Suppose $u \in M_{r}^{s}\left(\mathscr{K}_{\delta}\right)$. Then $u^{* *} \in M_{r}^{s}(\mathscr{F})$ and is a $\mathscr{K}_{\delta} \cap \mathscr{F}_{-}$ regular extension of $u$ to $\sigma(\mathscr{F})$.

Proof. That $u^{* *}$ is $\sigma$-smooth follows from the fact that $\mathscr{K}_{\delta} \cap \mathscr{F}$ is a compact lattice (any collection $\left\{f_{\alpha}\right\}$ from the lattice has a nonempty intersection whenever every finite subcollection has a nonempty intersection). The rest of the theorem follows from Corollary 4.2.

The following definition is useful in determining when $u^{* *}$ is countably additive.

Definition 4.7. $L$ countably allocates $K$ if whenever $k \subseteq \cup_{1}^{\infty} l$, then there exist $k_{i} \in K$ such that each $k_{i}$ is contained in a finite union of the $l_{j}$ and $\bigcup_{1}^{\infty} k_{i}=k$. If $L^{\prime}$ countably allocates $K$ then $L$ countably coallocates $K$.

THEOREM 4.8. Suppose $L$ countably coallocates $K$. Consider a countably additive measure $u$ on $\sigma(K)\left(\right.$ or $\left.R_{\sigma}(K)\right)$. Then $u_{*}$ is $\sigma$-smooth on $L^{\prime}$.

Proof. Suppose $l^{\prime}=\bigcup_{1}^{\infty} l_{j}^{\prime}$ and $\bigcup_{1}^{\infty} l_{j}^{\prime} \in L^{\prime}$. Choose $k \subseteq l^{\prime}$. There

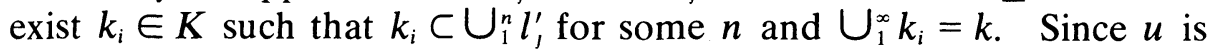
countably additive, $\lim _{i} u\left(k_{i}\right)=u(k)$. Thus $u_{*}\left(l^{\prime}\right) \leqq \lim _{n} u_{*}\left(\bigcup_{1}^{n} l_{\prime}^{\prime}\right)$. The reverse inequality is always true.

In a locally compact Hausdorff space if $k \subseteq \cup_{1}^{\infty} o_{j}$ where the $o_{j}$ are 
open, then $k=\bigcup_{1}^{n} k_{i}, k_{i} \in \mathscr{K}_{\delta}$, and $k_{i} \subseteq o_{J}$ for some $j$. Thus $\mathscr{F}$ countably coallocates $\mathscr{K}_{\delta}$. Also for every $k \in \mathscr{K}_{\delta} k \subseteq z_{1}^{\prime} \subseteq k_{1}$ where $z_{1}$ is a zero set and $k \in \mathscr{K}_{\delta}$. Applying Theorems 4.8, 3.3 and 3.5 we obtain the following.

THEOREM 4.9. Let $X$ be a locally compact Hausdorff space. Every countably additive measure $u$ on $R_{\sigma}\left(\mathscr{K}_{\delta}\right)$, is $\mathscr{K}_{\delta}$-inner regular. $u^{* *}$ is a countably additive extension of $u$ to $\sigma(\mathscr{F})$.

Proof. All that has to be shown is that $u$ is $\mathscr{K}_{\delta}$-inner regular. This follows from the fact that for each $b \in R\left(\mathscr{K}_{\delta}\right), b=\bigcup_{1}^{\infty} k_{j}, k_{j} \in \mathscr{K}_{\delta}$.

Levin and Stiles [8] showed that the conclusions of Theorem 4.9 no longer are true if $R_{\sigma}\left(\mathscr{K}_{\delta}\right)$ is replaced by $\sigma\left(\mathscr{K}_{\delta}\right)$ even if $X$ is locally compact and Hausdorff. Suppose $X$ is locally compact, paracompact and Hausdorff. Levin and Stiles prove that for any countably additive measure $u$ on $\sigma\left(\mathscr{K}_{\delta}\right) u(b)=\inf \left\{u(o) / b \subseteq o, o\right.$ open and $\left.o \in \sigma\left(\mathscr{K}_{\delta}\right)\right\}$. Thus if $u$ is also $\mathscr{K}_{\delta}$-inner regular then $u^{* *}$ must be a countably additive extension of $u$ to $\sigma(\mathscr{F})$ according to Theorem 3.3. This result is found in the paper of Levin and Stiles.

In a countably paracompact, normal space the lattice $\mathscr{F}$ countably coallocates $\mathscr{Z}$. In any topological space, for every zero set $z, z \subseteq z_{1}^{\prime} \subseteq z_{2}$ where $z_{1}, z_{2}$ are zero sets. Thus we obtain Marik's [9] result.

THEOREM 4.10. Every countably additive measure $u$ on $\sigma(\mathscr{Z})$ is $\mathscr{Z}$-inner regular. If $X$ is countably paracompact and normal then $u^{* *}$ is a countably additve extension of $u$ to $\sigma(\mathscr{F})$.

Let $X$ be a countable product, $\Pi_{1}^{\infty} X_{k}$, of discrete topological spaces. Define for $x=\left(x_{1}, \cdots\right), y=\left(y_{1}, \cdots\right) y=x(\bmod n)$ if $x_{i}=y_{i}$, $i=1, \cdots, n$. For any subset $A$ of $X$ define $t_{A}(x)$ to be the least positive integer $n$, if any, such that $y \in A$ whenever $y=x(\bmod n)$. If there exists no such $n$ then let $t_{A}(x)=+\infty$. Suppose $C \subseteq \cup_{1}^{\infty} O_{k}$ where $C$ is a clopen set (both closed and open in $X$ ) and each $O_{k}$ is open. Define inductively

$$
\begin{aligned}
& C_{1}=\left\{c \in C / t_{O_{1}}(c) \leqq t_{O_{k}}(c), k \neq 1\right\}, \\
& C_{n}=\left\{c \in C \backslash\left(\cup_{1}^{n-1} C_{i}\right) / t_{O_{n}}(c) \leqq t_{O_{k}}(c), k \neq n\right\} .
\end{aligned}
$$

Then $C=\cup_{1}^{\infty} C_{k}, C_{k} \subseteq O_{k}$ for all $k$ and each $C_{k}$ is clopen. Thus $\mathscr{F}$ countably coallocates $\mathscr{C} \ell$, the lattice of clopen sets. Dubins is interested 
in measures defined on $\mathscr{C} \ell=A(\mathscr{C} \ell)$. These measures are called strategic measures. Strategic measures are always $\mathscr{C} \ell$-inner regular.

THEOREM 4.11. Let $X$ be a countable product of discrete topological spaces. For every countably additive strategic measure $u, u^{* *}$ is a countably additive extension of $u$ to $\sigma(\mathscr{F})$.

Let $R$ be a ring of subsets in $X$. Define $\mathscr{L}(R)$ to be those subsets $b$ such that $b \cap r \in R$ for every $r \in R . \quad \mathscr{L}(R)$ is an algebra containing $R$. $\quad \mathscr{L}(R)$ certainly coallocates $R$ and if $R$ is a $\sigma$-ring then $\mathscr{L}(R)$ is an $\sigma$-algebra that countably coallocates $R$. For a measure (not necessarily finite valued on $R)$ define $u_{*}(b)=\sup \{u(r) / r \subseteq b, r \in R\}$, and $u^{* *}$ with respect to $\mathscr{L}(R)$. It is easy to see that $u^{* *}=u_{*}$ on $\mathscr{L}(R)$. By Theorem $3.3 u_{*}$ is an extension of $u$ to $\mathscr{L}(R)$. By Theorems 4.8 and 3.3 if $R$ is a $\sigma$-ring and $u$ is countably additive then $u_{*}$ is countably additive on $\mathscr{L}(R) . \quad \mathscr{L}(R)$ is called the class of sets locally measurable with respect to $R$. The result for countably additive measures on a $\sigma$-ring is found in a paper by Berberian [2].

If $K \subseteq L$ is an $L$-ideal, then $A(L) \subseteq \mathscr{L}(R(K))$. Clearly $l \cap r$ belongs to $R(K)$ for all $l \in L$ and $r \in R(K)$. Suppose $b \cap r$ and $c \cap r$ belong to $R(K)$ for all $r \in R(K)$. Then $(b \cup c) \cap r$ belongs to $R(K)$ for all $r \in R(K)$. If $b \cap r \in R(K)$ then $b^{\prime} \cup r^{\prime}$ is in $A(K)$. Therefore $r \cap b^{\prime}=r \cap\left(b^{\prime} \cup r^{\prime}\right)$ belongs to $R(K)$. Thus $A(L)$ is contained in $\mathscr{L}(R(K))$. Also $\sigma(L)$ is contained in $\mathscr{L}\left(R_{\sigma}(K)\right)$. Thus in a Hausdorff space $\sigma(\mathscr{F})$ is contained in the locally measurable sets of $R_{\sigma}(\mathscr{K})$ where $\mathscr{K}$ is the lattice of compact sets [Berberian and Jakobsen 3]. In a completely regular Hausdorff space $\sigma(\mathscr{Z})$ is contained in the locally measurable sets of $R\left(\mathscr{K}_{\delta}\right)$. We also have, for any lattice $K, A(K) \subseteq \mathscr{L}(R(K))$ and $\sigma(K) \subseteq \mathscr{L}\left(R_{\sigma}(K)\right)$. In the following theorems the measures need not be finite on any particular set.

THEOREM 4.12. Any measure on $R(K)$ extends to a $R(K)$ inner regular measure on $A(K)$. Any countably additive measure on $R_{\sigma}(K)$ extends to a $R_{\sigma}(K)$ inner regular, countably additive measure on $\sigma(K)$.

THEOREM 4.13. In a Hausdorff space any countably additive meas ure on $R_{\sigma}(\mathscr{K})$ has a countably additive, $R_{\sigma}(\mathscr{K})$ inner regular extension to $\sigma(\mathscr{F})$. In a completely regular Hausdorff space any countably additive measure on $R_{\sigma}\left(\mathscr{K}_{\delta}\right)$ can be extended to a countably additive, $R_{\sigma}\left(\mathscr{K}_{\delta}\right)$ inner regular measure on $\sigma(\mathscr{Z})$.

THEOREM 4.14. Let $K \subseteq L$ be a $L$-ideal. Then for every $R(K)$ inner regular measure on $A(K)$ has a $R(K)$ inner regular extension to 
$A(L) . \quad$ Every countably additive, $R_{\sigma}(K)$ inner regular measure on $\sigma(K)$ has a countably additive, $R_{\sigma}(K)$ inner regular extension to $\sigma(L)$.

In view of Theorem 4.14 the next example shows that coallocation is not necessary for every $K$ inner regular measure $u$ on $A(K)$ to have $u_{*}$ modular on $L^{\prime}$. Also countable coallocation is not implied if $u_{*}$ is $\sigma$-smooth on $L^{\prime}$ for every countably additive $K$ inner regular measure on $R_{\sigma}(K)$.

Topologize the set of real numbers as follows. For $x \neq 0$ or 2 a neighborhood basis for $x$ is the collection of open intervals containing $x$. A neighborhood basis for 0 is the collection of open intervals containing 0 and 1 . Likewise a neighborhood basis for 2 is the collection of open intervals containing 1 and 2 . The interval [0,2] is a compact closed set and the intervals $I_{1}=(-1,3 / 2)$ and $I_{2}=(1 / 2,3)$ are open sets. There does not exist a sequence $\left\{C_{n}\right\}$ of closed, compact sets such that $\cup_{n=1}^{\infty} C_{n}=[0,2]$ and each $C_{n}$ is contained in either $I_{1}$ or $I_{2}$. Therefore the closed sets $\mathscr{F}$ do not coallocate or countably coallocate the lattice of compact closed sets though this lattice is an $\mathscr{F}$-ideal.

5. The extension of $\tau$-smooth measures. A measure on a lattice $L$ is $\tau$-smooth if for any net $\left\{l_{\alpha}\right\}$ decreasing to $\varnothing, \lim _{\alpha} u\left(l_{\alpha}\right)=0$. We will study the measures on $A(L)$ which are $L$ inner regular, finite valued on $L$ and $\tau$-smooth on $L$. Denote these measures by $\mathcal{M}_{r}^{t}(L) . \quad M_{r}^{t}(L)$ are those measures in $\mathcal{M}_{r}^{t}(L)$ which are bounded.

For a lattice $L, \tau(L)$ is the smallest lattice containing $L$ that is closed under arbitrary intersections. We now show that every $u \in \mathcal{M}_{r}^{t}(L)$ extends to $u^{* *}$, defined with respect to $\tau(L)$ on $A(L)$, and $\tau$-smooth on $\tau(L)$.

Lemma 5.1. Let u be a measure on $A(L), \tau$-smooth on $L$. For any $t$ in $\tau(L)$,

$$
u_{*}\left(t^{\prime}\right)=\lim _{\alpha} u\left(l_{\alpha}^{\prime}\right)
$$

where $t^{\prime}=\cup_{\alpha} l_{\alpha}^{\prime}$ and $\left\{l_{\alpha}^{\prime}\right\}$ is an increasing net of sets from $L^{\prime}$.

Proof. Choose $l \subseteq t^{\prime}$. Since $t \in \tau(L)$ there exists a net $\left\{l_{\alpha}^{\prime}\right\}$ from $L^{\prime}$ which is increasing and $\bigcup_{\alpha} l_{\alpha}^{\prime}=t^{\prime}$. Since $u$ is $\tau$-smooth, $\lim _{\alpha} u\left(l_{\alpha}^{\prime}\right)=$ $u(l)+\lim _{\alpha} u\left(l_{\alpha}^{\prime} \cap l^{\prime}\right)$. Therefore $u_{*}\left(t^{\prime}\right)=\lim _{\alpha} u\left(l_{\alpha}^{\prime}\right)$.

THEOREM 5.2. Suppose $u$ is a measure on $A(L), \tau$-smooth on L. Then $u_{*}$ is modular on $\tau(L)^{\prime}$. 
Proof. Let $s, t \in \tau(L)$. Then $s^{\prime}=\bigcup_{\alpha} h_{\alpha}^{\prime}, t^{\prime}=\bigcup_{\beta} l_{\beta}^{\prime}$ where $\left\{h_{\alpha}^{\prime}\right\},\left\{l_{\beta}^{\prime}\right\}$ are increasing nets from $L^{\prime}$.

Form the net $\left\{k_{\gamma}^{\prime}\right\}$ of unions $k_{\gamma}^{\prime}=h_{\alpha}^{\prime} \cup l_{\beta}^{\prime}$. For the same $\gamma, \alpha$, and $\beta$ define $p_{\gamma}^{\prime}=h_{\alpha}^{\prime} \cap l_{\beta}^{\prime} . \quad\left\{k_{\gamma}^{\prime}\right\}$ is a net increasing to $t^{\prime} \cup s^{\prime}$ and $\left\{p_{\gamma}^{\prime}\right\}$ is a net increasing to $t^{\prime} \cap s^{\prime}$. Thus

$$
\begin{aligned}
u_{*}\left(t^{\prime} \cup s^{\prime}\right)+u_{*}\left(t^{\prime} \cap s^{\prime}\right) & =\lim _{\gamma}\left(u\left(k_{\gamma}^{\prime}\right)+u\left(p_{\gamma}^{\prime}\right)\right) \\
& =\lim _{\gamma}\left(u\left(h_{\alpha}^{\prime}\right)+u\left(l_{\beta}^{\prime}\right)\right) \\
& \leqq u_{*}\left(t^{\prime}\right)+u_{*}\left(s^{\prime}\right) .
\end{aligned}
$$

TheOREM 5.3. Let $u \in \mathcal{M}_{r}^{t}(L)$. If $u^{* *}$ is finite on $L$ then it extends $u$ to $A(\tau(L))$ and belongs to $\mathcal{M}_{r}^{t}(\tau(L))$.

Proof. $u^{* *}$ extends $u$ according to Theorems 5.2 and 3.4. $u^{* *}$ is $\tau$-smooth and finite on $\tau(L)$ since each $t \in \tau(L)$ is the intersection of sets from $L$. Consider $t, s$ from $\tau(L)$. Choose $v$ from $\tau(L)$ such that $s \subseteq v^{\prime}$ and $u^{* *}\left(v^{\prime}\right)-u^{* *}(s)<\epsilon$. Then $u^{* *}\left(t \cap s^{\prime}\right)-u^{* *}(t \cap v)<\epsilon$. Every set in $A(\tau(L))$ is of the form $\bigcup_{1}^{n} t_{k} \cap s_{k}^{\prime}$ where $s_{k}$ belongs to $\tau(L)$ and either $t_{k} \in \tau(L)$ or $t_{k}=X$. Therefore $u^{* *}$ is $\tau(L)$ inner regular.

COROLlARY 5.4. Let $u$ be a $L$ inner regular, countably additive measure on $R_{\sigma}(L), \tau$-smooth and finite on $L$. If $u^{* *}$ is finite on $L$ then $u^{* *}$ is a countably additive extension of $u$ to $\sigma(\tau(L))$ and $u^{* *}$ is $\tau$-smooth and finite on $\tau(L)$.

Corollary 5.5. Suppose $X$ is a completely regular space. Suppose $u$ is a $L$ inner regular, countably additive measure on $\sigma(\mathscr{Z})$ that is $\tau$-smooth and finite on $\mathscr{Z}$. Then $u^{* *}$ is a countably additive extension of $u$ to $\sigma(\mathscr{F})$ and $u^{* *}$ is $\tau$-smooth and finite on $\mathscr{F}$.

A collection of sets has the finite (countable) intersection property if every finite (countable) subcollection has a nonempty intersection. A lattice $L$ is compact if every collection with the finite intersection property has a nonempty intersection. $L$ is Lindelof if every collection with the countable intersection property has a nonempty intersection. A measure on a compact lattice is always $\tau$-smooth and any $\sigma$-smooth measure on a Lindelof lattice is $\tau$-smooth. $\mathcal{M}_{r}(L)$ are the $L$ inner regular measures on $A(L)$ that are finite on $L$ and $\mathcal{M}_{r}^{s}(L)$ are those that are also $\sigma$-smooth on $L$. 
COROLlaRY 5.6. If $L$ is compact then every $u \in M_{r}(L)$ for which $u^{* *}$ is finite on $L$ extends to $u^{* *} \in \mathcal{M}_{r}^{t}(\tau(L))$. If $L$ is Lindelof then for every $u \in \mathcal{M}_{r}^{s}(L)$ such that $u^{* *}$ is finite on $L, u^{* *} \in \mathcal{M}_{r}^{i}(\tau(L))$ and extends $u$.

The result concerning compact lattices has been proved by using Zorn's lemma to show that $u^{* *}$ on $A(\tau(L))$ is, in an appropriate sense, a maximal extension of $u$ [P. A. Meyer 10].

Suppose $u$ is a $L^{\prime}$ outer regular measure on $A(L)$. Then for any decreasing net $\left\{l_{\alpha}\right\}$ from $L$ such that $\bigcap_{\alpha} l_{\alpha} \in A(L), \lim _{\alpha} u\left(l_{\alpha}\right)=$ $u\left(\bigcap_{\alpha} l_{\alpha}\right)$. If $L$ is a regular lattice then this property is a sufficient condition for a measure $u$ to be $L^{\prime}$ outer regular.

Definition 5.7. $L$ is $K$ regular if for any $l \in L$ there exists $\left\{h_{\alpha}\right\}$ from $L$ such that $l=\bigcap_{\alpha} h_{\alpha}$ and for each $\alpha$ there exists $k_{\alpha}$ from $K$ such that $l_{\alpha} \subseteq k_{\alpha}^{\prime} \subseteq h_{\alpha}$. If $L=K$ then $L$ is a regular lattice.

THEOREM 5.8. Assume $L$ is $K$ regular and that $K \subseteq A(L)$. If for any net $\left\{l_{\alpha}\right\}$ decreasing to $\bigcap_{\alpha} l_{\alpha} \in A(L), \lim _{\alpha} u\left(l_{\alpha}\right)=u\left(\cap l_{\alpha}\right)$ then $u$ is $K^{\prime}$ outer regular on $L$. If $K=L$ then $u$ is $L^{\prime}$ outer regular on $A(L)$. In addition, if $u$ is finite on $L$ and $L$ is regular, then $u$ is $L$ inner regular on $A(L)$.

Proof. The collection $\left\{l_{\alpha}\right\} \subseteq L$ such that $l_{\alpha} \supseteq k_{\alpha}^{\prime} \supseteq l$, is a net decreasing to $l$. Therefore

$$
\begin{aligned}
u(l) & \leqq \inf \left\{u\left(k_{\alpha}^{\prime}\right) / l \subseteq k_{\alpha}^{\prime} \subseteq l_{\alpha}\right\} \\
& \leqq \inf \left\{u\left(l_{\alpha}\right) / l \subseteq k_{\alpha}^{\prime} \subseteq l_{\alpha}\right\} \\
& =u(l)
\end{aligned}
$$

To give a similar result for measures on $\sigma(L)$ we need the following theorem. $\quad \delta(L)$ is the smallest lattice containing $L$ closed under countable intersections.

THEOREM 5.9. Let $u$ be a countably additive, $\sigma$-finite measure on a ring $R$ containing $L$. If $u$ is $L$ inner regular then the countably additive extension of $u$ to $R_{\sigma}(R)$ is $\delta(L)$ inner regular.

Proof. Let $S$ be the collection of sets $s$ in $R_{\sigma}(R)$ such that $u(s)=\sup \{u(k) / l \subseteq s, \quad l \in \delta(L)\}$. Then $R \subseteq S$. Let $\left\{s_{k}\right\}$ be any sequence from $S$ such that $u\left(s_{k}\right)$ is finite for all $k$. Then since $u$ is countably additive, $\cup_{1}^{\infty} s_{k}$ and $\bigcap_{1}^{\infty} s_{k}$ belong to $S$. 
Take any set $b$ in $R_{\sigma}(R)$ such that $u(b)$ is finite. There exists a sequence $\left\{r_{k}\right\}$ from $R$ such that $r=\bigcup_{1}^{\infty} r_{k}$ contains $b$ and $u(r)-u(b)<$ $\epsilon$. There exists $\left\{t_{k}\right\}$ from $R$ such that $t=\bigcup_{1}^{\infty} t_{k}$ contains $r \backslash b$ and $u(t)<\epsilon$. Then $r \backslash t \subseteq b$ and $u(b)-u(r \backslash t)<\epsilon$. For each $k, r \backslash t_{k}=$ $\bigcup_{j=1}^{\infty} r_{j} \backslash t_{k}$ belongs to $S$. Since $r \backslash t=\bigcap_{1}^{\infty} r \backslash t_{k}, r \backslash t$ belongs to $S$. This implies that $b$ belongs to $S$.

Every $b \in R_{\sigma}(R)$ is the countable union of sets $b_{k}$ such that $u\left(b_{k}\right)$ is finite. Therefore $R_{\sigma}(R)=S$. A similar proof shows the extension of $u$ is $\delta(L)^{\prime}$ outer regular when $u$ is $L^{\prime}$ outer regular.

THEOREM 5.10. Suppose $L$ is a regular lattice. Let u be a countably additive, $\sigma$-finite measure on $\sigma(L)$, finite on $L$. If for any net $\left\{l_{\alpha}\right\}$ decreasing to $\bigcap_{\alpha} l_{\alpha} \in A(L), \lim _{\alpha} u\left(l_{\alpha}\right)=u\left(\bigcap_{\alpha} l_{\alpha}\right)$, then $u$ is $\delta(L)$ regular on $\sigma(L)$.

COROLlary 5.11. Let $X$ be a topological space and u a countably additive, finite measure defined on $\sigma(\mathscr{F})$ such that for any decreasing net of closed sets $\left\{f_{\alpha}\right\}$

$$
\lim _{\alpha} u\left(f_{\alpha}\right)=u\left(\bigcap_{\alpha} f_{\alpha}\right) .
$$

(i) If $X$ is a regular space then $u$ is $\mathscr{F}$ regular. closed set $f$

(ii) If $X$ is completely regular then $u$ is $\mathscr{F} \cap \mathscr{L}$-regular and for every

$$
u(f)=\inf \left\{u\left(z^{\prime}\right) / f \subseteq z^{\prime}, z \in \mathscr{Z}\right\} .
$$

(iii) If $X$ is 0 -dimensional then $u$ is $\mathscr{F} \cap \mathscr{C} \ell$ regular where $\mathscr{C} \ell$ is the lattice of clopen sets and for every closed set $f$

$$
u(f)=\inf \{u(c) / f \subseteq c, c \text { clopen }\}
$$

(iv) If $X$ is a locally compact Hausdorff space then $u$ is $\mathscr{K}_{\delta} \cap \mathscr{F}$ regular and for every closed set $f$

$$
u(f)=\inf \left\{u\left(k^{\prime}\right) / f \subseteq k^{\prime}, k \in \mathscr{K}_{\delta}\right\}
$$

Corollary 5.12. Suppose $X$ is a locally compact Hausdorff space and $u$ a countably additive, finite measure on $\sigma(\mathscr{Z})$ such that for any decreasing net $\left\{z_{\alpha}\right\}$ of zero sets, where $\bigcap_{\alpha} z_{\alpha} \in A(\mathscr{Z})$, 


$$
\lim _{\alpha} u\left(z_{\alpha}\right)=u\left(\bigcap_{\alpha} z_{\alpha}\right)
$$

Then $u$ is $\mathscr{K}_{\delta}$ regular.

\section{Part (i) of 5.11 was proven by Gardner [6].}

\section{REFERENCES}

1. A. D. Alexandroff, Additive set functions in abstract spaces, Mat. Sb., (N.S.) 8, 50 (1940) 307-348. Mat. Sb. (N.S.) 8, 50 (1941), 563-621.

2. S. K. Berberian, On the extension of Borel measures, Proc. Amer. Math. Soc., 16 (1965), $415-418$.

3. S. K. Berberian and J. F. Jakobsen, A note on Borel sets, Amer. Math. Soc. Month., 70 (1963), 55.

4. G. Birkhoff, Lattice Theory, New York, Amer. Math. Soc., 1948.

5. L. Dubins, On Lebesgue-like extensions of finitely additive measures, The Annals of Prob., 2 No.

3, (1974), 456-463.

6. R. J. Gardner, The regularity of Borel measures and Borel measure compactness, Proc. London Math. Soc., 3, 30 (1975), 95-113.

7. P. R. Halmos, Measure Theory, Van Nostrand, New York, 1950.

8. M. Levin and W. Stiles, On the regularity of measures on locally compact spaces, Proc. of the Amer. Math. Soc., 36, 1, (1972), 201-206.

9. J. Marik, The Baire and Borel measure, Czech. Math. J., 82 (1957), 248-253.

10. P. A. Meyer, Probability and Potentials, Blaisdell Publ. Comp. 1966.

11. R. Sikorski, Boolean Algebras, Springer-Verlag, New York, 1969.

12. F. Topsoe, Compactness in Spaces of Measures, Studia Math., 36 (1970), 195-212.

13. V. S. Varadarajan, Measures on topological spaces, Amer. Math. Soc. Translations, Series (2) 48 (1965), 161-228.

Received February 11, 1977.

Polytechnic Institute of New York

BROOKLYN, NY 11201

Current address: Mathematics Dept.

University of Wisconsin at Milwaukee

Milwaukee, WI 53211. 



\section{PACIFIC JOURNAL OF MATHEMATICS EDITORS}

ICHARD ARENS (Managing Editor)

niversity of California

os Angeles, CA 90024

\section{. A. BEAUmont}

niversity of Washington

sattle, WA 98105

. C. MOORE

niversity of California

erkeley, CA 94720
J. DUGUNDJI

Department of Mathematics

University of Southern California

Los Angeles, CA 90007

R. FINN AND J. MiLgRAM

Stanford University

Stanford, CA 94305

\section{ASSOCIATE EDITORS}
F. BECKENBACH
B. H. NEUMANN
F. WOLF
K. YosHidA

\section{SUPPORTING INSTITUTIONS}

NIVERSITY OF BRITISH COLUMBIA

UNIVERSITY OF SOUTHERN CALIFORNIA

ALIFORNIA INSTITUTE OF TECHNOLOGY STANFORD UNIVERSITY

NIVERSITY OF CALIFORNIA

ONTANA STATE UNIVERSITY

UNIVERSITY OF HAWAII

NIVERSITY OF NEVADA

UNIVERSITY OF TOKYO

EW MEXICO STATE UNIVERSITY

UNIVERSITY OF UTAH

REGON STATE UNIVERSITY

NIVERSITY OF OREGON

WASHINGTON STATE UNIVERSITY

UNIVERSITY OF WASHINGTON

SAKA UNIVERSITY

AMERICAN MATHEMATICAL SOCIETY

The Supporting Institutions listed above contribute to the cost of publication of this Journal, but they a t owners or publishers and have no responsibility for its contents or policies.

Mathematical papers intended for publication in the Pacific Journal of Mathematics should be in typ Irm or offset-reproduced (not dittoed), double spaced with large margins. Underline Greek letters in re ierman in green, and script in blue. The first paragraph or two must be capable of being used separately as 'nopsis of the entire paper. Items of the bibliography should not be cited there unless absolutely necessary, hich case they must be identified by author and Journal, rather than by item number. Manuscripts, uplicate, may be sent to any one of the four editors. Please classify according to the scheme of Math. Revieu Idex to Vol. 39. All other communications should be addressed to the managing editor, or Elaine Bart Iniversity of California, Los Angeles, California, 90024.

100 reprints are provided free for each article, only if page charges have been substantially pai dditional copies may be obtained at cost in multiples of 50 .

The Pacific Journal of Mathematics is issued monthly as of January 1966. Regular subscription rate: \$72. year (6 Vols., 12 issues). Special rate: $\$ 36.00$ a year to individual members of supporting institutions.

Subscriptions, orders for numbers issued in the last three calendar years, and changes of address should ent to Pacific Journal of Mathematics, 103 Highland Boulevard, Berkeley, California, 94708.

UBLISHED BY PACIFIC JOURNAL OF MATHEMATICS, A NON-PROFIT CORPORATION

Printed at Jerusalem Academic Press, POB 2390, Jerusalem, Israel.

Copyright (C) 1978 Pacific Journal of Mathematics

All Rights Reserved 


\section{Pacific Journal of Mathematics}

\section{Vol. 75, No. $1 \quad$ September, 1978}

Mieczyslaw Altman, General solvability theorems

Denise Amar and Eric Amar, Sur les suites d'interpolation en plusieurs variables ..........................................

Herbert Stanley Bear, Jr. and Gerald Norman Hile, Algebras which satisfy a second order linear partial differential equation ..................

Marilyn Breen, Sets in $R^{d}$ having $(d-2)$-dimensional kernels ............

Gavin Brown and William Moran, Analytic discs in the maximal ideal space

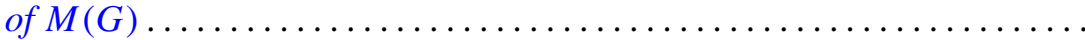

Ronald P. Brown, Quadratic forms with prescribed Stiefel-Whitney

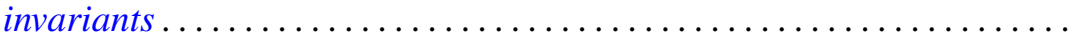

Gulbank D. Chakerian and H. Groemer, On coverings of Euclidean space by convex sets

S. Feigelstock and Z. Schlussel, Principal ideal and Noetherian groups.....

Ralph S. Freese and James Bryant Nation, Projective lattices ............

Harry Gingold, Uniqueness of linear boundary value problems for

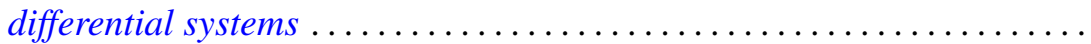

John R. Hedstrom and Evan Green Houston, Jr., Pseudo-valuation domains. . .

William Josephson, Coallocation between lattices with applications to measure extensions

M. Koskela, A characterization of non-negative matrix operators on $l^{p}$ to $l^{q}$ with $\infty>p \geq q>1$

Kurt Kreith and Charles Andrew Swanson, Conjugate points for nonlinear differential equations...........................

Shoji Kyuno, On prime gamma rings ........................ 185

Alois Andreas Lechicki, On bounded and subcontinuous multifunctions ..

Roberto Longo, A simple proof of the existence of modular automorphisms in approximately finite-dimensional von Neumann algebras ...

Kenneth Millett, Obstructions to pseudoisotopy implying isotopy for

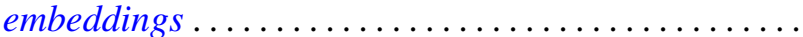

William F. Moss and John Piepenbrink, Positive solutions of elliptic equations. .

Mitsuru Nakai and Leo Sario, Duffin's function and Hadamard's

conjecture

Mohan S. Putcha, Word equations in some geometric semigroups ...

Walter Rudin, Peak-interpolation sets of class $C^{1} \ldots \ldots \ldots$

Elias Saab, On the Radon-Nikodým property in a class of locally convex

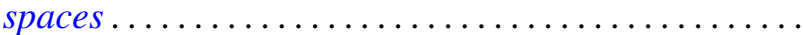

